\title{
Effect of Herbicides on Growth and Yield of Chickpea
}

\author{
Raghvendra Singh ${ }^{1 *}$, Ravi Shanker Singh ${ }^{1}$, R. K. Pathak ${ }^{2}$, \\ Kuldeep Singh ${ }^{1}$ and Vishal Singh ${ }^{1}$
}

\author{
${ }^{1}$ Department of Agronomy, ${ }^{2}$ Department of Soil Science and Agriculture Chemistry, Acharya \\ Narendra Deva University of Agriculture and Technology, Narendra Nagar (Kumarganj), \\ Ayodhya, India \\ *Corresponding author
}

\section{A B S T R A C T}

\section{Keywords}

Evaluation, imazamox, Pendimethalin etc.

\section{Article Info}

Accepted: 15 November 2020 Available Online: 10 December 2020
An investigation entitled "Evaluation of new herbicides and its combination for effective weed management in Chickpea (Cicer arietinum L.)" was carried out during Rabi season of year 2017-18 and 2018-19 at Agronomy Research Farm, Acharya Narendra Deva University of Agriculture and Technology. Kumarganj, Ayodhya (U.P.). Experiment was carried out with 12 treatments viz: $\mathrm{T}_{1^{-}}$Imazethapyr + imazamox (PRE), $\mathrm{T}_{2^{-}}$Imazethapyr+ imazamox (POE) at 3-4 leaf stage, $\mathrm{T}_{3^{-}}$ Imazethapyr (POE) at 3-4 leaf stage, $\mathrm{T}_{4}$-Quizalofop ethyl (POE) at 3-4 leaf stage, $\mathrm{T}_{5^{-}}$ Clodinofop (POE) at 3-4 leaf stage, $\mathrm{T}_{6}$-Pendimethalin (PE), $\mathrm{T}_{7}$-Pendimethalin (PE)+ Imazethapyr (POE), $\mathrm{T}_{8}$-Oxyfluorfen (PE), $\mathrm{T}_{9}$ - Oxyfluorfen (PE)+ Quizalofop (POE), $\mathrm{T}_{10-} 1$ Hand Weeding at 35-40 DAS, $\mathrm{T}_{11^{-}}$Weed Free and $\mathrm{T}_{12^{-}}$Weedy Check respectively laid out in Randomized Block Design with three replication. The objective of the study was to find out the effect of herbicides on growth and yield of chickpea. Different weed control treatments did not influence the number of plants $\mathrm{m}$ 2, plant height and dry weight of plants at 30th day stage of crop growth. At 60th, 90th and at harvest stages, post-emergence application of either Quizalofop or imazethapyr at $60 \mathrm{~g}$ ha-1, each applied in the pendimethalin $1000 \mathrm{~g}$ ha- 1 or oxyfluorfen $200 \mathrm{~g}$ ha-1 as pre-emergence treated plots (T7 \& T9) being at par recorded significantly higher plant height and crop dry matter over rest of the treatments including only the application of either pendimethalin $1000 \mathrm{~g}$ ha- 1 or oxyfluorfen $200 \mathrm{~g}$ ha-1 as preemergence alone (T6 and T7).

\section{Introduction}

Pulses are an important commodity group of crops that provide high quality protein complementing cereal proteins for predominantly substantial vegetarian population of the country. Although, being the largest pulse crop cultivating country in the world, the cultivation of pulses builds-up a mechanism to fix atmospheric nitrogen in their root nodules and thus meet their nitrogen requirements to a great extent. 
In India, pulses can be produced with a minimum use of resources and hence, pulses become less costly even than animal protein. As compared to vegetables, pulses are rich in protein which are less expensive and can be cultivated as sole crop, inter-crop and as mixed crop, as well. Pulses are mostly cultivated under rainfed conditions and do not require intensive irrigation facility and this is the reason that pulses are grown in areas left after satisfying the demand for cereals/cash crops.

Even in such conditions, pulses give better returns. Apart from this, pulses possess several other qualities such as they are rich in protein, improve soil fertility and physical structure, fit in mixed/inter-cropping system, crop rotations and dry farming and provide green pods for vegetable and nutritious fodder for cattle as well.

Introduction of herbicides has made it possible to control a wide spectrum of weeds in pulses effectively at a remunerative cost. Application of pendimethalin as pre-emergence at $1.0 \mathrm{~kg}$ ha $^{-1}$ (Tewari et al., 2003 and Vaishya et al., 2005), imazethapyr as post-emergence at 0.1 $\mathrm{kg} \mathrm{ha}{ }^{-1}$ (Singh et al., 2003), clodinafoppropargyl (Topic $15 \mathrm{WP}$ )as post-emergence at $0.03 \mathrm{~kg} \mathrm{ha}^{-1}$ (Marwat et al., 2004) and oxyfluorfen (600 $\mathrm{g} \mathrm{ha}^{-1}$ ) as weed control treatment (Yousefi et al., 2007) provided effective control of annual broad leaved and grassy weeds in chickpea field as reported by many research workers from the various parts of the country.

Weeds adversely affect the growth, yield and quality of crops by competing with them for space available soil moisture, nutrients and sunlight. Weeds indirectly reduce the yield potential by serving as alternate host to a number of crop pests. Weed species particularly, Vicia sativa in gram, provides shelter to Helicoverpa armigera, a major pest of chickpea. Pendimethalin is a selective dinitroaniline herbicide used to control most of the annual grasses and certain broadleaf weeds in maize, oil seeds, pulses and vegetables, etc. Imazethapyr, kills weeds by inhibiting the enzyme acetohydroxy acid synthase (AHAS), which is involved in the synthesis of three branches chain of aliphatic amino acids, leucine, isoleucine and valine. This inhibition causes a disruption in protein synthesis, which leads to an interference in DNA synthesis and cell growth.

\section{Materials and Methods}

The field experiment was conducted during rabi season of the year 2017-18 and 2018-19 at Agronomy Research Farm of Acharya Narenra Deva University of Agriculture and Technology, Narendra Nagar (Kumarganj), Ayodhya (U.P.) India. To find out the effect of weed control treatments on crop and associated weeds, to study the efficacy of pendimethalin and oxyfluorfen as preemergence in combination with postemergence herbicides. The experiment was carried out with 12 treatments viz: $\mathrm{T}_{1^{-}}$ Imazethapyr + imazamox(PRE), $\quad \mathrm{T}_{2}-$ Imazethapyr+ imazamox (POE) at 3-4 leaf stage, $\mathrm{T}_{3}$ - Imazethapyr (POE) at 3-4 leaf stage, $\mathrm{T}_{4}$-Quizalofop ethyl (POE) at 3-4 leaf stage, $\mathrm{T}_{5}$-Clodinofop (POE) at 3-4 leaf stage, $\mathrm{T}_{6^{-}}$ Pendimethalin(PE), $\mathrm{T}_{7}$-Pendimethalin $(\mathrm{PE})+$ Imazethapyr (POE), $\mathrm{T}_{8}$-Oxyfluorfen (PE), $\mathrm{T}_{9^{-}}$ Oxyfluorfen (PE)+ Quizalofop (POE), $\mathrm{T}_{10-} 1$ Hand Weeding at 35-40 DAS, $\mathrm{T}_{11}$ - Weed Free and $\mathrm{T}_{12^{-}}$Weedy Check respectively. The experiment was laid out in Randomized Block Design (RBD) with three replications.

\section{Results and Discussion}

Different weed control treatments did not influence the number of plants $\mathrm{m}^{-2}$, plant height and dry weight of plants at $30^{\text {th }}$ day stage of crop growth. 
Number of pods plants $\left(\mathrm{m}^{-2}\right)$

At 30 DAS stage of the chickpea, plant population was not affected significantly due to different weed control treatments. Only preemergence applied herbicides e.g. Imazethapyr + imazamox (PRE) (T1) and Pendimethalin (PE) (T6) did not show any phytotoxic effect on crop.

But later on post emergence application of herbicide at 35 DAS as a follow up application may be in single or mixed combination had the effect on plant growth and development.

As follow up applied on Pendimethalin(PE) + Imazethapyr (POE) (T7) recorded significantly higher plant population followed by Oxyfluorfen (PE) along with quizalofop 60 g. The same trend was observed at 90th day and at harvest stages also.

\section{Plant height}

At 30 DAS stage of crop growth, the plant height was not affected significantly due to weed control treatments. At 60th day stage, the highest plant height was recorded with $\mathrm{T} 7$ (26.52 and $26.80 \mathrm{~cm})$ fb T6 (25.70 and 26.00 $\mathrm{cm})$ and T9 $(25.50$ and $25.80 \mathrm{~cm})$; and the lowest with T4 $(23.20$ and $23.50 \mathrm{~cm})$, T5 (23.50 and $23.90 \mathrm{~cm})$ and T3 (24.00 and 24.40 $\mathrm{cm})$, respectively both years of experimentation.

At 60 DAS stage of crop growth, the plant height was not affected significantly due to weed control treatments. At 90th day stage, the highest plant height was recorded with T7 (35.90 and $36.20 \mathrm{~cm}$ ) fb T6 (35.10 and 35.40 $\mathrm{cm})$ and T9 $(34.90$ and $35.20 \mathrm{~cm})$; and the lowest with T4 $(32.60$ and $32.90 \mathrm{~cm}), \mathrm{T} \neg 5$ (32.90 and $33.30 \mathrm{~cm}$ ) and $\mathrm{T} \neg 3$ (33.40 and $33.80 \mathrm{~cm}$ ), respectively both years of experimentation. At harvest stage of crop growth, the plant height was not affected significantly due to weed control treatments. At harvest stage, the highest plant height was recorded with $\mathrm{T} 7(43.60$ and $43.90 \mathrm{~cm})$, T6 (42.80 and $43.40 \mathrm{~cm}$ ) and T9 (42.60 and 42.90 $\mathrm{cm})$; and the lowest with T4 (40.30 and 40.60 $\mathrm{cm}), \mathrm{T} \neg 5(40.60$ and $41.00 \mathrm{~cm})$ and $\mathrm{T} \neg 3$ (41.10 and $41.50 \mathrm{~cm})$, respectively both years of experimentation.

Treatment T7, T6 and T9 achieved the higher plant height might be because of the fact that both of these pre-emergence applied herbicides e.g. pendimethalin $1000 \mathrm{~g}$ ha-1 or oxyfluorfen $20 \mathrm{~g}$ ha-1 along with follow up application Imazethapyr or quizalofop $60 \mathrm{~g}$ ha- 1 each killed the broad and narrow leaved weeds very effectively, resulted lowest competition of various weed species with crop and ultimately found more plant height and likewise low plant height was recorded in T4: Quizalofop ethyl (POE), T5: Clodinofop (POE) and T3: Imazethapyr (POE), might be due to the fact that narrow leaved weeds escaped to a greater extent which caused the competition with crop and ultimately reduced the plant height at all the stages of crop growth.

\section{Dry mater accumulation of crop plant ${ }^{-1}$}

Crop dry matter is a net result of photosynthesis which remains in balanced after respiration process. At the same time, growth attributes e.g. plant height, number branches and plant population have the direct bearing in contributing the dry matter accumulation, while density and the dry weight of the weeds have a strongly negative correlation.

This is very true here also that the treatments, reduced the density and dry weight of the weeds more effectively, provided a more favourable micro-environment to enhance the crop growth and ultimately having more crop dry weight in the respective treatments. 
Crop dry matter accumulation was increased appreciably due to the different treatments as compared to the weedy check as all the growth stages of crop. Dry matter recorded at 30th day stage of the crop growth was not affected significantly due to different weed control treatments. Because early stage there was not much competition between crop and weed plants.

But at later stages, dry matter accumulation by crop was influenced to a greater extent due to different weed control treatments. At 60th day stage maximum dry matter accumulation with T7: Pendimethalin (PE) + Imazethapyr (POE) (4.60 and $4.70 \mathrm{~g}$ plant-1), T6: oxyfluorfen Pendimethalin(PE) (4.55 and $4.64 \mathrm{~g} \mathrm{plant}^{-1}$ ), T9: Oxyfluorfen (PE)+ Quizalofop (POE) (4.46 and $4.55 \mathrm{~g}$ plant $^{-1}$ ) and T8: p Oxyfluorfen (PE) (4.35 and $4.44 \mathrm{~g} \mathrm{plant}^{-1}$ ) both years of experimentation.

These all the treatments being at par recorded significantly higher dry weight as compared to T3: Imazethapyr (POE) (4.15 and $4.22 \mathrm{~g}$ plant-1) and T5: Clodinofop (POE) (4.00 and 4.10 g plant-1). While, T4: Quizalofop ethyl (POE) and T2: Imazethapyr + imazamox (POE) showed the dry mater at par with each other as well as found same trend followed by responsible growth stage. As far as the treatment combinations were concerned, they showed higher dry matter accumulation as compared to application of single treatment (T3, T4 and T5), in which crop was noticed. Combination of the treatment T7 showed effective control of grassy and BLWs also. However, in case of single herbicide e.g. pendimethalin or oxyfluorfen controlled both type of weed very effectively at early stage but weeds which emerged at later stages could compete with the crop and ultimately declined the crop dry weight and yield levels. On the other hand, the herbicides applied as a follow up application (PoE) controlled the later emerged weed species, though Imazethapyr and quizalofop $60 \mathrm{~g} \mathrm{ha}^{-1}$ each could controlled only Phalaris minor and Avena fatua as well as some other annual grassy weeds, while imazethapyr $75 \mathrm{~g} \mathrm{ha}^{-1}$ (PoE) controlled the BLWs especially Chenopidium album, Melilotus species, Vicia species and Convolvulus arvensis etc. resultant to which crop got a favourable weed free microenvironment and achieved the better growth and yield attributes and yield.

\section{Number of branches plant-1}

At 60th and 90th day stages of crop growth, weed free treatment did not show much difference as compared to T7: pendimethalin $1000 \mathrm{~g}$ (PE) along with Imazethapyr (PoE), T9: Oxyfluorfen (PE) + Quizalofop (POE), T6:Pendimethalin(PE), T8: Oxyfluorfen (PE) and T2 : Imazethapyr + imazamox (POE) being at par recorded significantly higher number of braches plant-1 as compared to weedy check, respectively both the years.

The similar trend was observed at 90th day sage also. This may be because of the fact that treatments which have better weed control efficiency caused more horizontal crop growth as a result produced more number of branches plant-1.

\section{Number of nodules plant $^{-1}$}

At 30th day stage of chickpea, significantly higher number of nodules plant-1 was recorded with weed free treatment both the year.

Among herbicide treatments e.g. T7: Pendimethalin (PE)+ Imazethapyr (POE) and T9: Oxyfluorfen (PE)+ Quizalofop (POE) being at par recorded significantly higher number of nodules plant-1 over alone treatment T2: Imazethapyr + imazamox followed by T1: Imazethapyr + imazamox (PRE) and Te: Imazethapyr (POE). 
Table.1 Effect of weed control treatments on plant height $(\mathrm{cm})$ at various growth stages of chickpea

\begin{tabular}{|c|c|c|c|c|c|c|c|c|}
\hline \multirow[t]{2}{*}{ Treatments } & \multicolumn{2}{|c|}{30 DAS } & \multicolumn{2}{|c|}{60 DAS } & \multicolumn{2}{|c|}{ 90DAS } & \multicolumn{2}{|c|}{ At harvest } \\
\hline & 2017-18 & 2018-2019 & 2017-18 & $\begin{array}{l}2018- \\
2019\end{array}$ & 2017-18 & $\begin{array}{c}2018 \\
2019\end{array}$ & 2017-18 & $\begin{array}{c}2018 \\
2019\end{array}$ \\
\hline Imazethapyr+ imazamox ( PRE) & 15.66 & 15.68 & 24.20 & 24.50 & 33.60 & 33.90 & 41.30 & 41.60 \\
\hline Imazethapyr+ imazamox ( POE) at 3-4 leaf stage & 15.70 & 15.72 & 24.40 & 24.70 & 33.80 & 34.10 & 41.50 & 41.80 \\
\hline Imazethapyr (POE) at 3-4 leaf stage & 15.62 & 15.64 & 24.00 & 24.40 & 33.40 & 33.80 & 41.10 & 41.50 \\
\hline Quizalofop ethyl ( POE) at 3-4 leaf stage & 15.55 & 15.57 & 23.20 & 23.50 & 32.60 & 32.90 & 40.30 & 40.60 \\
\hline Clodinofop (POE) at 3-4 leaf stage & 15.59 & 15.61 & 23.50 & 23.90 & 32.90 & 33.30 & 40.60 & 41.00 \\
\hline Pendimethalin(PE) & 16.00 & 16.02 & 25.70 & 26.00 & 35.10 & 35.40 & 42.80 & 43.10 \\
\hline Pendimethalin(PE)+ Imazethapyr(POE) & 16.20 & 16.22 & 26.50 & 26.80 & 35.90 & 36.20 & 43.60 & 43.90 \\
\hline Oxyfluorfen (PE) & 15.75 & 15.77 & 24.80 & 25.10 & 34.20 & 34.50 & 41.90 & 42.20 \\
\hline Oxyfluorfen (PE)+ Quizalofop (POE) & 15.90 & 15.92 & 25.50 & 25.80 & 34.90 & 35.20 & 42.60 & 42.90 \\
\hline 1 Hand Weeding at 35-40 DAS & 15.86 & 15.88 & 25.40 & 25.70 & 34.80 & 35.10 & 42.50 & 42.80 \\
\hline Weed Free & 16.40 & 16.42 & 28.80 & 29.20 & 38.20 & 38.60 & 46.90 & 47.30 \\
\hline Weedy Check & 15.40 & 15.42 & 20.10 & 20.50 & 29.50 & 29.90 & 35.20 & 35.60 \\
\hline SEm \pm & 0.70 & 0.69 & 1.00 & 1.09 & 1.27 & 1.13 & 1.78 & 1.68 \\
\hline C.D. at $5 \%$ & 2.07 & 2.03 & 2.93 & 3.19 & 3.73 & 3.32 & 5.23 & 4.92 \\
\hline
\end{tabular}


Table.2 Effect of weed control treatments on dry matter accumulation $\left(\mathrm{g} \mathrm{m}^{-2}\right)$ of various growth stages of chickpea

\begin{tabular}{|c|c|c|c|c|c|c|c|c|}
\hline \multirow[t]{2}{*}{ Treatments } & \multicolumn{2}{|c|}{30 DAS } & \multicolumn{2}{|c|}{60 DAS } & \multicolumn{2}{|c|}{ 90DAS } & \multicolumn{2}{|c|}{ At harvest } \\
\hline & 2017-18 & 2018-19 & 2017-18 & 2018-19 & 2017-18 & 2018-19 & 2017-18 & 2018-19 \\
\hline Imazethapyr+ imazamox ( PRE) & 1.48 & 1.51 & 4.21 & 4.28 & 8.00 & 8.15 & 16.40 & 16.70 \\
\hline Imazethapyr+ imazamox ( POE) at 3-4 leaf stage & 1.50 & 1.53 & 4.25 & 4.33 & 8.10 & 8.26 & 16.60 & 16.95 \\
\hline Imazethapyr ( POE) at 3-4 leaf stage & 1.47 & 1.50 & 4.15 & 4.22 & 7.90 & 8.06 & 16.20 & 16.50 \\
\hline Quizalofop ethyl ( POE) at 3-4 leaf stage & 1.46 & 1.49 & 3.90 & 3.98 & 7.41 & 7.55 & 15.20 & 15.47 \\
\hline Clodinofop ( POE) at 3-4 leaf stage & 1.48 & 1.51 & 4.00 & 4.10 & 7.60 & 7.75 & 15.60 & 15.90 \\
\hline Pendimethalin (PE) & 1.52 & 1.55 & 4.55 & 4.64 & 8.65 & 8.81 & 17.75 & 18.10 \\
\hline Pendimethalin(PE)+ Imazethapyr(POE) & 1.54 & 1.57 & 4.60 & 4.70 & 8.75 & 8.92 & 17.94 & 18.30 \\
\hline Oxyfluorfen (PE) & 1.50 & 1.53 & 4.35 & 4.44 & 8.25 & 8.42 & 16.91 & 17.26 \\
\hline Oxyfluorfen (PE)+ Quizalofop ( POE) & 1.51 & 1.54 & 4.46 & 4.55 & 8.48 & 8.65 & 17.38 & 17.74 \\
\hline 1 Hand Weeding at 35-40 DAS & 1.51 & 1.54 & 4.44 & 4.53 & 8.45 & 8.61 & 17.32 & 17.65 \\
\hline Weed Free & 1.56 & 1.60 & 4.71 & 4.81 & 9.06 & 9.25 & 18.56 & 18.96 \\
\hline Weedy Check & 1.45 & 1.46 & 2.80 & 2.85 & 4.62 & 4.71 & 10.86 & 11.10 \\
\hline SEm \pm & 0.06 & 0.06 & 0.17 & 0.14 & 0.27 & 0.28 & 0.62 & 0.66 \\
\hline C.D. at $5 \%$ & 0.17 & 0.19 & 0.50 & 0.42 & 0.78 & 0.82 & 1.82 & 1.93 \\
\hline
\end{tabular}


Table.3 Effect of weed control treatments on number of branches plant ${ }^{-1}$ of various growth stages of chickpea

\begin{tabular}{|c|c|c|c|c|}
\hline Treatments & \multicolumn{2}{|c|}{ 60 DAS } & \multicolumn{2}{c|}{ 90DAS } \\
\hline & $\mathbf{2 0 1 7 - 1 8}$ & $\mathbf{2 0 1 8 - 2 0 1 9}$ & $\mathbf{2 0 1 7 - 1 8}$ & $\mathbf{2 0 1 8 - 2 0 1 9}$ \\
\hline Imazethapyr+ imazamox ( PRE) & 10.10 & 10.30 & 20.60 & 20.80 \\
\hline Imazethapyr+ imazamox ( POE) at 3-4 leaf stage & 10.30 & 10.50 & 21.10 & 21.20 \\
\hline Imazethapyr ( POE) at 3-4 leaf stage & 9.90 & 10.10 & 20.20 & 20.50 \\
\hline Quizalofop ethyl ( POE) at 3-4 leaf stage & 9.40 & 9.55 & 19.20 & 19.40 \\
\hline Clodinofop ( POE) at 3-4 leaf stage & 9.60 & 9.75 & 19.60 & 19.80 \\
\hline Pendimethalin(PE) & 10.90 & 11.05 & 22.20 & 22.50 \\
\hline Pendimethalin(PE)+ Imazethapyr(POE) & 11.00 & 11.15 & 22.50 & 22.70 \\
\hline Oxyfluorfen (PE) & 10.50 & 10.65 & 21.40 & 21.60 \\
\hline Oxyfluorfen (PE)+ Quizalofop ( POE) & 10.70 & 10.85 & 21.80 & 22.00 \\
\hline 1 Hand Weeding at 35-40 DAS & 10.65 & 10.80 & 21.70 & 21.90 \\
\hline Weed Free & 11.35 & 11.50 & 23.10 & 23.50 \\
\hline Weedy Check & 7.75 & 7.90 & 15.80 & 16.10 \\
\hline SEm & 0.46 & 0.45 & 0.87 & 0.99 \\
\hline C.D. at 5\% & 1.36 & 1.32 & 2.54 & 2.89 \\
\hline
\end{tabular}


Table.4 Effect of weed control treatments on number of nodules of various growth stages of chickpea

\begin{tabular}{|c|c|c|c|c|c|c|}
\hline \multirow[t]{2}{*}{ Treatments } & \multicolumn{2}{|c|}{30 DAS } & \multicolumn{2}{|c|}{60 DAS } & \multicolumn{2}{|c|}{ 90DAS } \\
\hline & 2017-18 & 2018-19 & 2017-18 & 2018-19 & 2017-18 & 2018-19 \\
\hline Imazethapyr + imazamox (POE) at 3-4 leaf stage & 9.35 & 9.40 & 17.00 & 17.10 & 11.80 & 11.88 \\
\hline Quizalofop ethyl (POE) at 3-4 leaf stage & 8.56 & 8.71 & 15.57 & 15.84 & 10.81 & 11.00 \\
\hline Clodinofop (POE) at 3-4 leaf stage & 8.64 & 8.87 & 15.70 & 16.12 & 10.90 & 11.20 \\
\hline Pendimethalin (PE) & 10.06 & 10.20 & 18.30 & 18.52 & 12.20 & 12.35 \\
\hline Oxyfluorfen (PE)+ Quizalofop ( POE) & 9.98 & 10.10 & 18.15 & 18.37 & 12.10 & 12.25 \\
\hline 1 Hand Weeding at 35-40 DAS & 9.94 & 10.05 & 18.10 & 18.30 & 12.05 & 12.20 \\
\hline Weed Free & 10.55 & 10.68 & 19.20 & 19.40 & 12.45 & 12.60 \\
\hline Weedy Check & 7.40 & 7.52 & 13.45 & 13.65 & 9.20 & 9.35 \\
\hline SEm \pm & 0.39 & 0.41 & 0.63 & 0.61 & 0.48 & 0.49 \\
\hline
\end{tabular}


Table.5 Effect of weed control treatments on fresh weight of nodules plant ${ }^{-1}(\mathrm{mg})$ at different growth stages of chickpea

\begin{tabular}{|c|c|c|c|c|c|c|}
\hline \multirow[t]{3}{*}{ Treatments } & \multicolumn{6}{|c|}{ Fresh weight of nodules plant ${ }^{-1}$} \\
\hline & \multicolumn{2}{|c|}{30 DAS } & \multicolumn{2}{|c|}{60 DAS } & \multicolumn{2}{|c|}{90 DAS } \\
\hline & 2017-18 & 2018-19 & 2017-18 & 2018-19 & 2017-18 & 2018-19 \\
\hline Imazethapyr+ imazamox ( PRE) & 689.13 & 696.58 & 1283.97 & 1295.40 & 821.34 & 828.36 \\
\hline $\begin{array}{l}\text { Imazethapyr + imazamox (POE) at 3-4 leaf } \\
\text { stage }\end{array}$ & 696.58 & 700.30 & 1295.40 & 1303.02 & 828.36 & 833.98 \\
\hline Imazethapyr (POE) at 3-4 leaf stage & 685.40 & 693.60 & 1274.83 & 1289.30 & 815.72 & 824.85 \\
\hline Quizalofop ethyl (POE) at 3-4 leaf stage & 637.72 & 648.90 & 1186.43 & 1207.01 & 758.86 & 772.20 \\
\hline Clodinofop (POE) at 3-4 leaf stage & 643.68 & 660.82 & 1196.34 & 1228.34 & 765.18 & 786.24 \\
\hline Pendimethalin (PE) & 749.47 & 759.90 & 1394.46 & 1411.22 & 856.44 & 866.97 \\
\hline Pendimethalin (PE)+ Imazethapyr (POE) & 756.18 & 763.63 & 1405.89 & 1419.61 & 863.46 & 871.88 \\
\hline Oxyfluorfen (PE) & 737.55 & 745.00 & 1371.60 & 1388.36 & 842.40 & 852.93 \\
\hline Oxyfluorfen (PE)+ Quizalofop (POE) & 743.51 & 752.45 & 1383.03 & 1399.79 & 849.42 & 859.95 \\
\hline 1 Hand Weeding at 35-40 DAS & 740.53 & 748.73 & 1379.22 & 1394.46 & 845.91 & 856.44 \\
\hline Weed Free & 785.98 & 795.66 & 1463.04 & 1478.28 & 873.99 & 884.52 \\
\hline Weedy Check & 551.30 & 560.24 & 1024.89 & 1040.13 & 645.84 & 656.37 \\
\hline SEm \pm & 25.16 & 33.30 & 52.90 & 6.38 & 35.15 & 30.87 \\
\hline C.D. at $5 \%$ & 73.79 & 97.66 & 155.14 & 185.89 & 103.08 & 90.53 \\
\hline
\end{tabular}


Table.6 Effect of weed control treatments on dry weight of nodules plant ${ }^{-1}(\mathrm{mg})$ at different growth stages of chickpea

\begin{tabular}{|c|c|c|c|c|c|c|}
\hline \multirow[t]{3}{*}{ Treatments } & \multicolumn{6}{|c|}{ Dry weight of nodules plant ${ }^{-1}$} \\
\hline & \multicolumn{2}{|c|}{30 DAS } & \multicolumn{2}{|c|}{60 DAS } & \multicolumn{2}{|c|}{90 DAS } \\
\hline & 2017-18 & 2018-19 & 2017-18 & 2018-19 & 2017-18 & 2018-19 \\
\hline Imazethapyr+ imazamox ( PRE) & 220.52 & 222.90 & 475.07 & 479.30 & 303.90 & 306.49 \\
\hline $\begin{array}{c}\text { Imazethapyr+ imazamox (POE) at 3-4 leaf } \\
\text { stage }\end{array}$ & 222.90 & 224.10 & 479.30 & 482.12 & 306.49 & 308.57 \\
\hline Imazethapyr (POE) at 3-4 leaf stage & 219.33 & 221.95 & 471.69 & 477.04 & 301.82 & 305.19 \\
\hline Quizalofop ethyl (POE) at 3-4 leaf stage & 204.07 & 207.65 & 438.98 & 446.59 & 280.78 & 285.71 \\
\hline Clodinofop (POE) at 3-4 leaf stage & 205.98 & 211.46 & 442.65 & 454.49 & 283.12 & 290.91 \\
\hline Pendimethalin (PE) & 239.83 & 243.17 & 515.95 & 522.15 & 316.88 & 320.78 \\
\hline Pendimethalin(PE)+ Imazethapyr (POE) & 241.98 & 244.36 & 520.18 & 525.25 & 319.48 & 322.60 \\
\hline Oxyfluorfen (PE) & 236.02 & 238.40 & 507.49 & 513.69 & 311.69 & 315.58 \\
\hline Oxyfluorfen (PE)+ Quizalofop (POE) & 237.92 & 240.78 & 511.72 & 517.92 & 314.29 & 318.18 \\
\hline 1 Hand Weeding at 35-40 DAS & 236.97 & 239.59 & 510.31 & 515.95 & 312.99 & 316.88 \\
\hline Weed Free & 251.51 & 254.61 & 541.32 & 546.96 & 323.38 & 327.27 \\
\hline Weedy Check & 176.42 & 179.28 & 379.21 & 384.85 & 238.96 & 242.86 \\
\hline SEm \pm & 10.80 & 10.13 & 19.44 & 19.72 & 12.54 & 12.12 \\
\hline C.D. at 5\% & 31.68 & 29.70 & 57.01 & 57.84 & 36.78 & 35.54 \\
\hline
\end{tabular}


Table.7 Effect of weed control treatments on yield contributing characters of chickpea

\begin{tabular}{|c|c|c|c|c|}
\hline \multirow[t]{2}{*}{ Treatments } & \multicolumn{2}{|c|}{ Number of pods plant ${ }^{-1}$ : } & \multicolumn{2}{|c|}{ Test weight } \\
\hline & 2017-18 & 2018-19 & 2017-18 & 2018-19 \\
\hline Imazethapyr+ imazamox ( PRE) & 30.80 & 31.00 & 17.59 & 17.43 \\
\hline Imazethapyr+ imazamox ( POE) at 3-4 leaf stage & 32.10 & 32.60 & 17.31 & 16.80 \\
\hline Imazethapyr ( POE) at 3-4 leaf stage & 29.00 & 29.30 & 17.33 & 17.70 \\
\hline Quizalofop ethyl ( POE) at 3-4 leaf stage & 25.80 & 26.20 & 16.85 & 17.86 \\
\hline Clodinofop ( POE) at 3-4 leaf stage & 27.60 & 28.10 & 17.29 & 16.79 \\
\hline Pendimethalin(PE) & 38.30 & 38.70 & 17.81 & 16.30 \\
\hline Pendimethalin(PE)+ Imazethapyr(POE) & 39.50 & 40.20 & 18.62 & 17.33 \\
\hline Oxyfluorfen (PE) & 34.60 & 35.00 & 16.79 & 16.50 \\
\hline Oxyfluorfen (PE)+ Quizalofop ( POE) & 36.50 & 37.00 & 17.82 & 16.10 \\
\hline 1 Hand Weeding at 35-40 DAS & 36.20 & 36.40 & 16.99 & 18.50 \\
\hline Weed Free & 40.50 & 41.60 & 18.70 & 17.30 \\
\hline Weedy Check & 22.50 & 23.40 & 17.82 & 16.10 \\
\hline SEm \pm & 1.22 & 1.47 & 0.17 & 0.19 \\
\hline C.D. at $5 \%$ & 3.58 & 4.31 & NS & NS \\
\hline
\end{tabular}


Table.8 Effect of weed control treatments on yield contributing characters of chickpea

\begin{tabular}{|c|c|c|c|c|c|c|}
\hline \multirow[t]{2}{*}{ Treatments } & \multicolumn{2}{|c|}{ Grain yield (q/ha) } & \multicolumn{2}{|c|}{ Straw yield (q/ha) } & \multicolumn{2}{|c|}{ Harvest index } \\
\hline & 2017-18 & 2018-19 & 2017-18 & 2018-19 & 2017-18 & 2018-19 \\
\hline Imazethapyr + imazamox (PRE) & 15.30 & 15.45 & 24.48 & 24.55 & 36.55 & 36.58 \\
\hline Imazethapyr+ imazamox ( POE) at 3-4 leaf stage & 14.10 & 14.16 & 24.79 & 25.02 & 36.58 & 36.61 \\
\hline Imazethapyr (POE) at 3-4 leaf stage & 13.85 & 13.96 & 24.07 & 24.24 & 36.52 & 36.54 \\
\hline Quizalofop ethyl ( POE) at 3-4 leaf stage & 13.70 & 13.80 & 22.81 & 23.13 & 36.48 & 36.51 \\
\hline Clodinofop (POE) at 3-4 leaf stage & 13.10 & 13.30 & 23.83 & 23.98 & 36.50 & 36.53 \\
\hline Pendimethalin(PE) & 15.25 & 15.38 & 27.60 & 27.92 & 36.70 & 36.72 \\
\hline Pendimethalin (PE)+ Imazethapyr(POE) & 16.90 & 16.98 & 29.06 & 29.16 & 36.77 & 36.80 \\
\hline Oxyfluorfen (PE) & 14.30 & 14.45 & 25.86 & 25.92 & 36.63 & 36.66 \\
\hline Oxyfluorfen (PE)+ Quizalofop (POE) & 16.00 & 16.20 & 26.42 & 26.65 & 36.67 & 36.70 \\
\hline 1 Hand Weeding at 35-40 DAS & 14.95 & 15.00 & 26.35 & 26.55 & 36.66 & 36.68 \\
\hline Weed Free & 17.40 & 17.55 & 29.88 & 30.06 & 36.80 & 33.86 \\
\hline Weedy Check & 9.35 & 9.42 & 18.35 & 18.45 & 33.75 & 33.80 \\
\hline SEm \pm & 0.57 & 0.62 & 1.04 & 1.13 & 1.36 & 1.56 \\
\hline
\end{tabular}


However, T4: Quizalofop ethyl (POE) and T5: Clodinofop (POE) at par with each other both the year.

However, number of nodules plant-1 recorded lower in case of Quizalofop ethyl (POE) (T4) and Clodinofop (POE) (T5) which were at par recorded with other treatments. Likewise, Imazethapyr + imazamox (PRE). While in case of better weed control treatments, weed free condition provided profused root development and bacterial colonies, which ultimately resulted in more nodulation in crop. Almost similar trend was recorded at 60th day stage also both years of experimentation.

In case of 60th and 90th day stages, declined the number of nodules plant ${ }^{-1}$, respectively due to cessation of nodulation and started drying of nodules. The results are in agreement with the finding of Vaishya et al., (1995).

\section{Fresh and dry weight of nodules plant ${ }^{-1}$}

At 60th day stage of chickpea significantly higher fresh and dry weight of nodules plant-1 was recorded with weed free treatment. Among herbicide treatment T7: Pendimethalin (PE)+ Imazethapyr (POE) and T9: Oxyfluorfen (PE)+ Quizalofop (POE) being at par recorded significantly higher weight fresh and dry nodules plant-1 over alone applied herbicide. The similar trend was recorded with treatment T6, T8, T2 and T1. However, T2: Imazethapyr + imazamox (POE) and $\mathrm{T}^{-1}$ : Imazethapyr + imazamox (PRE) were at par with each other. While, lower fresh and dry weight of nodules plant-1 was recorded with Pendimethalin(PE)+ Imazethapyr (T7) and Oxyfluorfen (PE)+ Quizalofop (POE) (T9) being at par recorded with other treatments T6 and T8). With respect a combination of either quizalofop or clodinafop at $60 \mathrm{~g} \mathrm{ha}^{-1}$ each tank mixed with oxyfluorfen $200 \mathrm{~g} \mathrm{ha}^{-1}$ applied as post-emergence. Almost similar trend was recorded at 90th day stage also with respect to fresh and dry weight of nodules plant ${ }^{-1}$.

\section{Yield attributes}

The different yield contributing characters e.g. number of pods plant ${ }^{-1}$ and test weight were influenced significantly due to different weed control treatments. The treatment T7: Pendimethalin(PE)+ Imazethapyr (POE), T9: Oxyfluorfen (PE)+ Quizalofop (POE), T6: Pendimethalin (PE), T8: Oxyfluorfen (PE) alone, T1: Imazethapyr+ imazamox (PRE) and T2: Imazethapyr+ imazamox (POE) being at par recorded significantly higher value of number of pods plant ${ }^{-1}$ over T3: Imazethapyr (POE), T4: Quizalofop ethyl (POE) and T5: Clodinofop (POE) respectively. However, lowest and highest values of number of pods plant- 1 were recorded with weedy and weed free treatment, respectively both the year.

As far as the 100-grain weight or test weight of chickpea was concerned, it was not affected significantly due to different weed control treatments as it is directly related with genetic characters of the crop or a variety. However, higher test weight was recorded with T7: Pendimethalin (PE) + Imazethapyr (POE) followed by T9: Oxyfluorfen (PE)+ Quizalofop (POE). Butter et al., (2008) also reported the similar type of response of weed control treatments.

\section{References}

Marwat, K.B.; Khan, Hanif and Zahid, I.A. (2004). Efficacy of different herbicides for controlling grassy weeds in chickpea (Cicer arietinum L.). Pakistan Journal of Weed Science Research,10(314): 139-143.

Singh, R.N.; Sharma, A.K. and Tomar, R.K.S. (2003). Weed control in chickpea under late sown condition. Indian Journal of Agronomy, 48(2): 114-116. 
Tewari, A.N.; Tewari, S.N.; Rathi, J.P.S.; Singh, B. and Tripathi, A.K. (2003). Effect of cultural and chemical methods on weed growth and grain yield of dwarf pea. Indian Journal of Weed Science,35(1\&2): 49-52.

Vaishya, R.D.; Quaizad, M.F.; Singh, S. and Rajput, A.L. (1995). Effect of seed rate and weed management practices on nodulation and yield of chickpea.
Indian J. of Agron. 40(2): 314-315. Vaishya, R.D.; Fayaz, M. and Srivastava,V.K. (2005). Integrated weed management in chickpea. Indian Journal. Agronomy., 9: 34-98.

Yousefi, Alizadeh, A.R.; Rahimian, H.M.H. (2007). Broad leaf weed control in chickpea (Cicer arietinum L.) with pre and post-emergence herbicides. Research on Crops, 8(3): 560-564.

\section{How to cite this article:}

Raghvendra Singh, Ravi Shanker Singh, R. K. Pathak, Kuldeep Singh and Vishal Singh. 2020. Effect of Herbicides on Growth and Yield of Chickpea. Int.J.Curr.Microbiol.App.Sci. 9(12): 3479-3492. doi: https://doi.org/10.20546/ijcmas.2020.912.413 